\title{
Viajar en el espacio-tiempo: Física y literatura en la historia de tu vida
}

\author{
Travel in spacetime: Physics and literature in story of your life
}

\author{
PANAGIOTIS Xouplidis ${ }^{1}$
}

Como citar este artículo: Xouplidis, P. (2021). Viajar en el espacio-tiempo: Física y literatura en la historia de tu vida, Pangeas. Revista Interdisciplinar de Ecocrítica (núm. 3) 16-25. https://doi.org/10.14198/ PANGEAS.18862

\begin{abstract}
Resumen
Todo tipo de viaje es función del espacio y del tiempo. Según la teoría de la relatividad de Einstein, espacio y tiempo pasan a ser dimensiones de una nueva entidad (el espacio-tiempo) y Bajtín introduce el concepto del cronotopo (tiempo-espacio) para expresar el carácter indisoluble del espacio y el tiempo en la literatura. El premiado relato Story of Your Life (La historia de tu vida) de Ted Chiang narra el encuentro de la lingüista Dra. Banks con los heptápodos, seres extraterrestres que llegan a la Tierra en sus naves espaciales. La protagonista se enfrenta con una distinta visión del mundo a través del lenguaje alienígena donde lo enunciado determina el tiempo vivido. El relato introduce la posibilidad de un viaje en el tiempo mediante un lenguaje extraterrestre que conceptualiza la vida en el espacio-tiempo sin los límites humanos. Basada en las interconexiones entre el lenguaje, el tiempo y el espacio, la narración revisa el mundo objetivo y revela lo arbitrario de nuestra percepción del tiempo. Desde el punto de vista de teorías lingüísticas y literarias complementadas por conceptos de Física y Matemáticas, el artículo ofrece una aproximación al viaje en el tiempo a través del texto de ciencia ficción de Ted Chiang.
\end{abstract}

Palabras clave: Espacio-tiempo; lenguaje; máquina de tiempo; ciencia ficción; Ted Chiang.

\section{Abstract}

Every type of journey is a function of space and time. According to Einstein's theory of relativity, space and time become dimensions of a new entity space-time and Bajtin introduces the concept of chronotope (time-space) to express the indissoluble character of space and time in literature. Ted Chiang's award-winning Story of Your Life chronicles linguist Dra.Banks' encounter with the heptapods, extraterrestrial beings arriving on Earth on their spaceships. The protagonist is confronted with a different view of the world through the alien language where the statement determines the time lived. The story introduces the possibility of a journey in time through extraterrestrial spoken language that conceptualizes life in space-time without human limits. Based on the interconnections between language, time and space, the narrative reviews the objective world and reveals the arbitrary of our perception of time. From the point of view of linguistic and literary theories complemented by elements of physics and mathematics, the article offers an approximation of time travel through Ted Chiang's science fiction.

Key words: Spacetime; language; time-machine; science fiction; Ted Chiang.

1. Panagiotis Xouplidis. AristotleUniversity of Thessaloniki, Greece. pxouplid@gmail.com 


\section{INTRODUCCIÓN}

Si es posible el viaje en el tiempo, el pasado y el futuro existen. Si no fuera así, sería imposible llegar a un destino inexistente dado que todo tipo de viaje es función del espacio y del tiempo. En las culturas humanas muchas veces el punto de partida del tiempo es el principio de las narraciones fundamentales, de los mitos esenciales de la civilización que los genera. Para los griegos antiguos, navegadores intrépidos del Mediterráneo, Ulises constituye el prototipo del viajero y con él empieza el tiempo de los viajes. En el viaje de retorno de Ulises se pone de manifiesto la progresiva individuación del héroe y el desarrollo de su disciplina racionalista (Boetsch, 2003: 57). Un viaje realizado por un héroe por un espacio vasto, y potencialmente desconocido, durante un periodo de tiempo se podría considerar una odisea, por lo tanto, los libros de Jules Verne "podríamos [ser considerados] una odisea" (Rodríguez Pequeño 2018:2). Verne reinventa la fórmula antigua de literatura de viajes incorporando la ciencia como elemento clave en el desarrollo de la historia, se atreve a introducir situaciones imposibles tratadas por la ciencia de su época. De este modo:

\begin{abstract}
La ciencia y el viaje son, junto con el héroe, los elementos principales. Podemos llamarlos odiseas científicas, porque en realidad son aventuras que giran alrededor de un viaje en el que la ciencia y/o la tecnología juegan un papel principal, puesto que el protagonista es un científico que, como Ulises, supera los obstáculos gracias a su inteligencia y a sus conocimientos científico-tecnológicos (Rodríguez Pequeño 2018:2).
\end{abstract}

El acceso al mundo físico es posible gracias a las experiencias del 'yo', en consecuencia, no experimentamos la realidad externa, sino nuestra inserción en ella (Zukav, 1981). En Física, la ilusión de la dicotomía entre el espacio y el tiempo ha sido destruida por la teoría de la relatividad, teoría cuyo efecto tendría implicaciones operativas en todo el concepto convencional de la realidad, por lo tanto, no resultaría extraño que en los estudios literarios el principio del espacio-tiempo se trasladara a la textualidad. Por ejemplo, la ficción de las novelas de Verne siempre está construida sobre una base científica sólida, hecho que dota a sus narraciones con un alto grado de verosimilitud dando los primeros pasos hacia un nuevo género: la ciencia ficción. Se podría afirmar que la literatura de ciencia ficción crea este espacio literario donde la ciencia interactúa con la literatura tratando siempre de no alejarse de la realidad del lector que se introduce en la ciencia por la literatura.

El premiado relato de ciencia ficción Story of Your Life (La historia de tu vida) de Ted Chiang narra el encuentro de dos especies que poseen modelos comunicativos disímiles, los humanos y los extraterrestres que llegan a la Tierra en sus naves espaciales. Basada en las interconexiones entre el lenguaje, el tiempo y el espacio, la narración desnuda nuestra condición de sujeto, revisa el mundo objetivo y revela lo arbitrario de nuestra percepción del tiempo. Desde el punto de vista de teorías lingüísticas y literarias complementadas por conceptos de Física y Matemáticas, el artículo ofrece una aproximación al viaje en el tiempo a través del texto de ciencia ficción de Ted Chiang.

\section{EL VIAJE EN EL TIEMPO EN LA HISTORIA DE TU VIDA}

Para muchos estudiosos, Edgar Allan Poe fue precursor del género que se definiría más tarde (en 1926) con el término "ciencia ficción". Unos relatos escritos por él casi un siglo antes podrían dar una primera idea sobre la relación entre la ciencia y la literatura:

[...], la ciencia ficción de Poe rara vez toma la ciencia misma, o la interrelación entre la ciencia y la sociedad, como tema. Las historias relacionadas con el proceso físico tienden a centrarse en la tecnología, como los detalles de los viajes aéreos, o técnicas, como los pases hipnóticos, en lugar de en la ciencia teórica o práctica (Franklin 1995: 88).

Por otra parte, Verne construye su obra a partir de "la narración fantástica verosímil" (Rodríguez Pequeño 2018: 12-13), caracterizada por una imaginación creadora que encuentra sus límites en la verosimilitud. Utiliza la ciencia para hacer verosímil lo fantástico, creando así 
una ficción literaria en la que lo fantástico está explicado mediante un principio científico. Narra situaciones imaginarias que se presentan como reales por teorías y medios científicos porque "precisamente tiene la misión de que esa fantasía parezca realizable, posible, verosímil" (Rodríguez Pequeño 2018:15).

En las obras de literatura de ciencia ficción el lector debe enfrentarse a alienígenas, tiempos futuros, planetas lejanos, sociedades e instituciones imaginarias fuera de su experiencia accesible que resultan disconformes con su realidad y que forman parte de los mundos de la ciencia ficción llamados "nova" (Rodríguez Hillón, 2015: 182). Durante la lectura de sus obras, los autores piden al lector que trate de comprender que todo lo que se considera posible y real obedezca a una configuración cultural y espaciotemporal. La opción al mundo literario, que la aproximación lectora a la ciencia ficción ofrece, se halla en su contrato ficcional con la realidad científica que no es la vivida por el lector sino la que se parece posible gracias a las teorías científicas en un futuro, lógicamente próximo, al contrario de otras narrativas. La ciencia ficción es un género que rompe las normas temporales, convirtiendo la narrativa en viaje a mundos posibles futuros.

Cabe mencionar que en la obra con el título El anacronópete (1887) el autor español Enrique Gaspar imagina y describe por primera vez una máquina del tiempo que permitía a los personajes de su novela desplazarse a cualquier momento del pasado. No obstante, dada la escasa difusión del texto, según la historia de la literatura oficial, diez años antes de la introducción del concepto acuñado por Einstein, la idea del viaje en el tiempo surge por primera vez con la difusión adecuada, en 1895, gracias a la novela de HG. Wells, La máquina del tiempo, que trata al tiempo como una cuarta dimensión en la que: "El hombre... puede ascender en contra de la gravedad en un globo. ¿Por qué no esperar ser capaz de detener o acelerar su viaje a través de la dimensión tiempo o, incluso, virar y viajar en sentido contrario?" (Wells, 2001: 7)

La novela pertenece al género de la literatura fantástica, un modo narrativo proveniente del realismo, aunque supone una transgresión de la realidad: los elementos literarios de dicho género parten de la verosimilitud que ofrece al lector la narración realista para introducirle a la irrupción del acontecimiento inexplicable, un enfoque esencial que se establece entre lo realista y lo fantástico (Roas 2001: 27). No obstante, aunque Verne añade en temáticas literarias ya existentes, principalmente en las novelas y relatos de viajes y de aventuras, la base científica, Wells, con ciertos elementos tecnológicos y científicos, se centra en la cuestión social y cultural que la tecnología podría ofrecer al hombre al darle la posibilidad de viajar por la línea temporal en una máquina de tiempo.

Cabe observar que todo tipo de viaje, cualesquiera sean sus características, está inscrito en lo temporal y su estructura se despliega en el tiempo. De la Física se sabe que una variante clave de cualquier viaje es la velocidad, función del espacio y del tiempo (De Oto \& Rodríguez, 2010: 11). Por consiguiente, la narrativa de ciencia ficción que imagina un viaje en el tiempo emplea unos recursos cuyas configuraciones se podrían tratar en función de un relato de viaje; se trata de un relato híbrido, la narración de un viaje ficcional sobre una base teórica científica. Entra así en el terreno de la literatura de viajes, aunque no sigue sus normas en el pacto ficcional por alterar el un desplazamiento convencional por el mundo real.

Se podría afirmar que la literatura de viajes tiene un carácter doble, descriptivo y narrativo a la vez; está permanentemente en tensión entre lo referencial y lo autorreferencial, por eso está considerado un género de frontera, un género que crece conviviendo con otros géneros en una frontera de movimiento continuo. Esta ubicación en los límites de otros géneros la convierte en "espacio de contacto" (Boetsch 2003-2005: 49) en los bordes mismos del sistema literario. Además, el viaje es la historia del cambio que sufre el sujeto de la narración, cuya función es expresar el contacto con la alteridad. Todo relato de viajes tiene estructuras narrativas organizadoras del espacio, por lo tanto "el espacio representado en el texto se vuelve un topos, es decir, una construcción imaginaria de un lugar" (Colombi Nicolia, 2006: 21-22). En el viaje el sujeto de la enunciación coincide con el sujeto del enunciado, remite a una instancia extratextual que indica quien escribe. Es el 'observador-viajero', cuya reflexión aparece en el texto en formato de notas o diario personal, en la escritura realizada durante 
el viaje. Este pacto afecta a la realidad del lector ante la perspectiva común entre autor, narrador y personaje, "el estudio del viaje nos coloca frente a la oposición literal y figurativo, referencial y no referencial, fáctico y ficcional, debatidas por las distintas teorías narrativas (Colombi Nicolia, 2006: 34-35)."

Cabe añadir que Borges opinaba que el viajero del tiempo sería un observador omnisciente (Parra, 1988), dado que la conciencia conceptualiza la vida como una imagen fugaz en el espacio que cambia continuamente en el tiempo, a través del lenguaje el sujeto "crea" la imagen del mundo (Weyl, 1949). En Física, según la teoría de la relatividad de Einstein, espacio y tiempo pasan a ser dimensiones de una nueva entidad (el espacio-tiempo) y, para Minkowsky el tiempo depende de la situación del movimiento de cada observador (Udías Vallina, 2014: 26). En la teoría literaria, "se requiere cierto equilibrio entre el sujeto observador y el objeto observado" porque "por un lado, es la ciencia; por el otro, la autobiografía; el 'relato de viaje' vive de la interpenetración de los dos" (Todorov, 1993: 101). En los libros de viaje la narrativa autobiográfica emerge del encuentro del hablante con información distante o poco familiar para reclamar validez literal a través de una constante referencia a la actualidad (Fussell, 1980: 203). Además, la narración autobiográfica es un "relato retrospectivo en prosa que una persona real hace de su propia existencia, poniendo énfasis en su vida individual y, en particular, en la historia de su personalidad" (Lejeune 1991: 48).

El encuentro entre unos alienígenas y una lingüista forma la base de La historia de tu vida (Story of Your Life) de Ted Chiang, un relato de ciencia ficción publicado en 1998. Los temas tratados son: la comunicación con seres extraterrestres, el lenguaje y el libre albedrío mediante las ideas del relativismo lingüístico de la hipótesis de Sapir-Whorf. La historia está narrada por la lingüista Dra. Louise Banks, la cual contratan las fuerzas militares para poder descifrar el idioma en que se comunican una especie de extraterrestres, conocidos como heptápodos (debido a su apariencia simétrica con siete extremidades). La historia se centra en Banks y su relación con el físico Gary Donnelly, que intenta obtener conocimientos de Física de los alienígenas. Los heptápodos tienen dos formas distintas de lenguaje: Heptápodo A es su lenguaje "oral", que se describe como idioma perfomativo no secuencial de orden libre de palabras y muchos niveles interpretativos; Heptápodo B, lenguaje "visual-escrito" que tiene una estructura totalmente distinta del lenguaje escrito humano. El conocimiento del lenguaje de los heptápodos afecta la manera de percepción del tiempo de la Dra. Banks y le introduce a la consciencia del mundo heptápodo, donde la causa y el resultado de los eventos se conocen simultáneamente:

Los humanos habían desarrollado un modo
de consciencia secuencial, mientras que
los heptápodos habían desarrollado un
modo de consciencia simultáneo. Nosotros
experimentábamos los acontecimientos en un
orden, y percibíamos la relación entre ellos como
causa y efecto. Ellos experimentaban todos los
acontecimientosa la vez, y percibían una intención
que los subyacía. Una intención minimizadora y
maximizadora (Chiang 2017: 112-113).

Se podría considerar que el concepto del tiempo parece ser una relación entre ciertos cambios (eventos) en cosas y un estado de conciencia que los registra. Si no hubiese seres conscientes en el universo, probablemente no habría tiempo. Parece que sus propiedades surgen de la interacción de un organismo dotado de conciencia con un mundo donde hay eventos externos a esa conciencia. El tiempo no es un simple instrumento estructural sino algo que el mismo lenguaje exige, una necesidad funcional obligatoria para la misma existencia del relato. En la literatura lo fantástico parece inscrito permanentemente en la realidad textual, pero a la vez se arremete contra esa misma realidad que lo enmarca en lo temporal.

La diégesis del relato de Chiang se organiza en el nivel diegético donde la acción da cuenta de la investigación del lenguaje heptápodo por Banks, y en el nivel metadiegético a modo de carta o diario dirigida a la hija de la lingüista donde se cuenta retrospectivamente la vida de esta misma hasta su concepción. Aunque en tiempos diferentes y en continuidad cronológica opuesta, ambas narraciones están en relación directa de acción y de espacio. Todo el metarrelato podría haberse considerado un viaje en el tiempo 
en relación con el relato de la investigación porque la acción desarrollada en el relato tiene su continuidad en el nivel metadiegético. Al establecerse esa continuidad, resulta como si el viaje en el tiempo del metarrelato proviniera del mundo del relato, produciendo una síntesis entre ambos: la presencia del tiempo curvo que permite el acceso a ambas narraciones. Los dos niveles del cuento se ocupan de desarrollar una historia diferente en espacios distintos unidos por un continuum temporal que se establece entre ambos desarrollando paralelamente secuencias narrativas entrecruzadas por el tiempo del discurso sobre el lenguaje en el relato y a través del lenguaje en el metarrelato.

En la literatura fantástica, uno de los recursos para viajar en el tiempo, además de las máquinas del tiempo, es la transferencia mental. De este modo, sin medio tecnológico, mediante los Ilamados sistemas de transferencia mental, un individuo es capaz de viajar mentalmente de modo prospectivo (ver el futuro) y/o retrospectivo (ver lo que sucedió en el pasado) y efectuar cambios en la línea temporal. Los hablantes de una lengua usan categorías que están disponibles en su lengua, por eso no sucede que el hablante no pueda ver una realidad obvia, sino que solamente sugiere asociaciones que son recibidas por la experiencia (Hickman 1987: 73). De ser así, todos los observadores se enfrentan con una distinta visión del mundo a través de los mismos hechos físicos, si su fondo lingüístico no puede reducirse a un denominador común. Parece que, "vemos, oímos y experimentamos como hacemos porque los hábitos lingüísticos de nuestra comunidad nos predisponen hacia ciertas elecciones interpretativas (Sapir, 1949: 69)." Además, "[...] el mundo se presenta en un flujo calidoscópico de impresiones que se deben organizar en nuestras mentes. Esto significa, principalmente, por los sistemas lingüísticos en nuestras mentes" (Whorf 1956: 212).

En el relato de Chiang la protagonista, la lingüista Dra. Banks, decide investigar el singular sistema ideográfico de escritura de los heptápodos y desarrolla un modelo de equivalencias aproximadas. La investigación de Banks plantea el concepto de semagrama:

En el siguiente informe que envié, planteé que el término "logograma" no era adecuado porque sugería que cada gráfico representaba una palabra hablada, cuando de hecho los gráficos no se correspondían con nuestra idea de palabras en absoluto. No quería tampoco usar en su lugar el término "ideograma" por cómo había sido usado en el pasado; propuse el término "semagrama" en su lugar (Chiang, 2017: 95).

Según la versión radical, la lengua determinaría toda la visión del mundo, es como una prisión; uno podría aspirar a comprender otro mundo, pero no sería posible porque la conciencia está formada por la lengua nativa de uno (Agar, 1994: 67). La versión débil, el relativismo lingüístico, indica que la lengua no determina el pensamiento sino exclusivamente lo conduce. Según el relativismo lingüístico, las lenguas afectan a nuestro modo de pensar en diferentes maneras.

En el relato de Chang, investigando el modelo comunicativo disímil de los extraterrestres, Banks llega a entender las convenciones sobre las que se asienta nuestra experiencia de lo real:

Para los heptápodos, todo el lenguaje era
performativo. En lugar de usar el lenguaje para
informar, usaban el lenguaje para realizar. Por
supuesto, los heptápodos ya sabían lo que se diría
en una conversación cualquiera; pero para que
su conocimiento se hiciera cierto, la conversación
tendría que suceder (Chiang 2017: 116).

Lo fantástico se vincula a menudo en sus definiciones con la expresión de un desorden, acecha una vuelta al caos primordial debida a la intervención de lo inexplicable, "se alimenta de una ruptura de lo verosímil que permite la entrada a lo inverosímil" (González Salvador, 1984: 215-216). Para los seres humanos, la conceptualización del tiempo depende de cómo los idiomas que ellos hablan tienden a hablar del tiempo, el contexto lingüístico y las metáforas particulares que suelen usarse para hablar sobre el tiempo en el momento de la comunicación. Según los resultados de relevantes estudios, conceptos fundamentales como el tiempo se diferencian entre culturas y revelan los mecanismos mediante los cuales el lenguaje y la cultura construyen las nociones básicas del tiempo (Boroditsky, 2011). Las personas bilingües parecen tener cierta flexibilidad sobre el concepto de la duración dependiendo del contexto lingüístico, ya que piensan de manera diferente en 
cuanto al tiempo, según el idioma usado (Fulga, 2012). El cambio de código lingüístico les permite organizaciones diferentes de conceptos básicos, como el tiempo; por ejemplo, suecos e ingleses normalmente se refieren a la duración utilizando analogías espaciales (distancia), mientras griegos y españoles suelen utilizar analogías cuantitativas. Por eso, suecos e ingleses perciben el tiempo como una línea, mientras griegos y españoles lo perciben como volumen. El tiempo se codifica en lenguaje normalmente a través de metáforas especificadas y condicionadas culturalmente (Casasanto et al., 2004). Parece que, "[...] nuestro cerebro tiene un mecanismo integrador que compone secuencias de eventos en el contenido de la conciencia, haciéndolos parecer para nosotros como el presente. [...] El ahora, el presente subjetivo, no es algo en forma independiente, sino un atributo de la conciencia" (Poeppel, 1978). Según la experiencia contada por Banks:

\section{Después de aprender heptápodo B, nuevos recuerdos aparecieron como bloques gigantes, cada uno abarcando años enteros, y aunque no llegaron en orden ni aterrizaron uno junto a otro, pronto compusieron un período de cinco décadas. Es el período durante el que conozco lo suficiente el heptápodo B para pensar en él, comenzando con mis entrevistas con Aleteo y Pedorreta y terminando con mi muerte (Chiang 2017:118).}

En el relato de Chiang, la historia, escrita en tiempo presente y en segunda persona, se cuenta a la hija de Banks, cuyo lugar ocupa el lector del texto: "Recuerdo una conversación que tendremos cuando estés en el primer año del instituto. Será un domingo por la mañana, y yo estaré batiendo unos huevos mientras tú pones la mesa para desayunar. Te estarás riendo mientras me cuentas acerca de la fiesta a la que fuiste anoche" (Chiang, 2017: 92). Banks narra en primera persona y en tiempo pasado la historia del encuentro con los heptápodos, mientras se intercalan las secciones que cuentan la vida de la hija a la misma, narrada como recuerdos de Banks escritos en tiempo futuro, dado que el conocimiento de Heptápodo B le permite a Banks conocer la vida entera de su hija antes de que ella fuera concebida: "Recuerdo cómo será mirarte cuando tengas sólo un día. Tu padre se habrá ido un momento a la cafetería del hospital, y tú estarás en tu cuna y yo me inclinaré sobre ti" (Chiang, 2017: 120).

Hablar del tiempo narrativo significa descodificar cómo el narrador construye un enunciado, cuyas dimensiones histórico-cronológicas y psicológicas del tiempo configuran el relato y enmarcan el desarrollo de las acciones de los personajes a través de la expresión lingüística. Las escuelas narratológicas han ofrecido distintas propuestas sobre la configuración del tiempo narrativo: Todorov diferencia entre tiempo del relato, tiempo de la escritura y tiempo de la lectura; Genette propone la distinción entre el tiempo de la historia, el tiempo del relato y el tiempo de la narración; Ricoeur distingue el tiempo prefigurado de la mimesis I (el tiempo real del autor), el tiempo configurado de la mimesis II (el tiempo organizado en la obra), y el tiempo refigurado de la mimesis III (el del lector que lo reinterpreta) (Contursi \& Ferro, 2000). Parece que la temporalidad determinante de la experiencia humana fomenta la relevancia del tiempo porque la relación entre los signos y el yo, entre texto y lector, se alcanza por medio del lenguaje, es decir, discursivamente. El concepto del cronotopo (tiempo-espacio) para expresar el carácter indisoluble del espacio y el tiempo en la literatura significa "conexión esencial de relaciones temporales y espaciales asimiladas artísticamente en la literatura" (Bajtín1989:237) que "determina la unidad artística de la obra literaria en sus relaciones con la realidad" (Bajtín, 1989: 393). La literatura está impregnada de "valores cronotópicos" (Bajtín 1989: 394) diversos, que se corresponden con cada motivo o elemento importante de la obra literaria. Este término de las ciencias matemáticas ha sido introducido y fundamentado a través de la teoría de la relatividad de Einstein y expresa el carácter indisoluble del espacio y el tiempo (el tiempo como la cuarta dimensión del espacio), mientras el cronotopo es una categoría de la forma y del contenido en la literatura (Bajtín, 1989). Especialmente, en el "cronotopo del camino" predomina el matiz temporal, ya que, en las novelas, por lo general, los encuentros se producen en el camino, un camino espacial y temporal en el que se cruzan las series espacio-temporales de las vidas y destinos humanos. En el cronotopo del camino, el tiempo se desarrolla sobre el espacio, "el tiempo es siempre el elemento central en las metaforizaciones del camino" (Bajtin, 1989: 408). El viaje en el tiempo de Banks abre el camino de 
la vida a la hija de Banks (La Historia de tu vida, según el título), cronotopo que se establece en el relato de Chiang. En la narración el espacio-tiempo se concibe como un continuum que no transcurre, el fluir temporal es un espejismo de la mente, convirtiendo el sueño de viajar por el tiempo en posibles caminos que se realizan en lenguaje. De ser así, el hecho de que el tiempo no tuviera una existencia absoluta se convertiría en una realidad lingüística si un sujeto pudiera sobrepasar los límites temporales convencionales a través de una narración. Si tuviésemos que concretar la presencia de la relatividad en el viaje por el espacio-tiempo en lenguaje humano y heptápodo, ésta estaría señalada por la performatividad narrativa (narrarviajar) del primero y la lingüística (hablar-actuar) del segundo. Cada una de éstas da lugar a los dos niveles diegéticos del relato: en el primero, el tiempo fluye en dirección al pasado y presenta una duración convencional (el conocimiento del lenguaje heptápodo) y en el segundo, el tiempo fluye del pasado y le permite a la Dra. Banks contarle a su hija el pasado en tiempo futuro, dado que la relatividad permite que el tiempo sea curvo para que ella viva una vida nueva ya contada.

Se podría deducir que el programa narrativo del relato sigue un esquema parecido al de uroboros. El uroboros - (del griego oupoßópoc [öpıc], '[serpiente] que se come la cola', a su vez de oủpó, "cola", y ßópoc, "que come") - es un símbolo que muestra a un animal serpentiforme que engulle su propia cola y que conforma, con su cuerpo, una forma circular. Representación de la inmovilidad perfecta y del movimiento perfecto que se eleva al Infinito - donde se asientan el principio del fin y el fin del principio -, representa la naturaleza cíclica de las cosas, el retorno eterno y otros conceptos percibidos como ciclos que comienzan de nuevo en cuanto concluyen. Además, simboliza el tiempo y la continuidad de la vida siendo representación de las cosas que cambian eternamente sin movimiento aparente alguno. A través de esta configuración de forma y contenido del relato, progresivamente, todo está sujeto a una reformulación del sentido real y figurado, un mundo suspendido por el lenguaje en la unidad del tiempo-espacio. La protagonista refuta su condición de sujeto, reivindica la armonía y la simetría como elementos constitutivos ante la revelación de lo arbitrario de su temporalidad. Banks se convierte en la observadora omnisciente de su propia vida futura y la de su hija, y se lo cuenta todo a ella (y al lector). El encuentro con los heptápodos le abre las puertas hacia el camino de su vida:

Trabajar con los heptápodos cambió mi vida. Conocí a tu padre y aprendí heptápodo B, y ambas cosas hacen posible que te conozca ahora, aquí en el patio a la luz de la luna. [...] Desde el comienzo sabía cuál era mi destino, y elegí el camino de acuerdo con él. [...] Y me separo de él, y nos tomamos de la mano mientras entramos en la casa para hacer el amor, para hacerte a ti (Chiang, 2017: 121).

Así la historia de la vida se convierte en camino hacia el destino conocido, el encuentro del espacio con el tiempo conduce la conciencia mediante el lenguaje a la memoria del futuro o la conciencia abre la memoria al futuro mediante el lenguaje. Así, toda historia es memoria en tiempo futuro, el bucle temporal hace accesibles todos los 'bloques' temporales simultáneamente en la conciencia lingüística de Banks: "[...] mi conciencia se convierte en una piedra de ámbar de medio siglo de longitud que arde fuera del tiempo. Percibo, durante esos atisbos, toda esta época como una simultaneidad. Es un período que cubre el resto de mi vida, y toda la tuya" (Chiang, 2017: 118).

La convivencia conflictiva de lo posible y lo imposible en términos de la realidad espaciotemporal del lector define este relato de ciencia ficción. En el conflicto que se produce, evidentemente, comparamos con la concepción que tenemos del tiempo real, lo temporalmente imposible no puede ser, no puede ocurrir porque permanece inexplicable según dicha concepción. Este conflicto determina una de las condiciones esenciales del funcionamiento de las obras de ciencia ficción: los acontecimientos deben desarrollarse en un mundo como el nuestro, construido en la idea temporal que tenemos de lo real, sin que este sea nuestro tiempo.

La teoría especial y general de la relatividad de Einstein estableció la visión del tiempo y el espacio como "estructuras maleables cuya forma y modo de presentarse dependen del estado de movimiento del observador" (Greene, 2006: 19). Así que tuvimos que abandonar el mundo newtoniano de las certezas y encontrarnos en un 
mundo donde no hay realidades universalmente válidas y percibidas de forma idéntica por todos los individuos. Parece que, "[...] la naturaleza, tal como la entendemos hoy, se comporta de tal modo que es fundamentalmente imposible hacer una predicción precisa de qué sucederá exactamente en un experimento dado" (Feynman, 2002: 67).

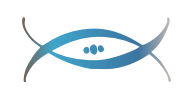

\section{CONCLUSIONES}

El lector de ciencia ficción, aunque reconoce su realidad en lo que lee, al mismo tiempo no la puede concebir como propia porque esta le señala posibilidades que le obligan a cuestionar su mundo, le ofrece los medios para explotar lo real investigando los fundamentos del tiempo presente y su desarrollo futuro (Moreno, 2009: 78-79). El lenguaje puede liberarse de los constreñimientos de la realidad para crear entidades completamente fantásticas carentes de existencia (Nandorfy, 2001: 250-251).

Wittgenstein dijo respecto a las revolucionarias ideas sobre el tiempo que surgieron a partir de los nuevos conceptos de la Física: "la única tarea que le queda a la filosofía es el análisis del lenguaje" (De Pienda, 2007: 267). Tanto el lenguaje escrito como el de las Matemáticas son sistemas específicos de símbolos que, aparte de su dimensión técnica, representan ideas que trascienden su significado. En el lenguaje escrito, las palabras representan sonidos, estableciendo un sistema de signos basado en asociaciones entre entidades del mundo. En el lenguaje matemático usado en la Física, los símbolos matemáticos representan conceptos del mundo científico. En ambos lenguajes, el uso continuo está directamente conectado con las entidades del mundo y las relaciones entre ellos que intentan representar. Aunque otros seres vivos se comunican entre sí, el lenguaje creativo que interpreta proyecta y evoluciona ideas pertenece exclusivamente a los seres humanos. Para los humanos, las palabras son ideas codificadas en sonidos y símbolos. Dotada con tal uso de lenguaje, la conciencia humana trasciende los límites de tiempo y espacio superando el mundo accesible a la percepción. Por lo tanto, no es el lenguaje como forma de comunicación lo que nos distingue de otros seres vivos, sino nuestra capacidad de crear un mundo de ideas mediante el lenguaje. Aunque existe una correlación arbitraria entre el significado en el mundo de las ideas y las entidades del mundo real, el lenguaje humano es la prueba de cómo se representan las ideas, articulándolas en la construcción del significado. La literatura amplifica esta capacidad para crear cronotopos, unidades espacio-temporales que el lector percibe como espacio-tiempo experimentado durante la lectura Desde el punto de vista de la Física: "Cuando combinamos la mecánica cuántica con la relatividad general parece haber una nueva posibilidad que no surgió antes: el espacio y el tiempo juntos podrían formar un espacio de cuatro dimensiones finito, sin singularidades ni fronteras, como la superficie de la Tierra, pero con más dimensiones" (Hawking, 1988: 164).

Con el objetivo de transcender lo real, sin abolir lo verosímil y centrada en su intento de representar lo imposible como científicamente posible, la narrativa de ciencia ficción se establece en el territorio fronterizo entre el lenguaje literario y la realidad experimentada. Por una parte, el lenguaje se nutre de la realidad, dado que la comprensión por parte del lector se basa en lo real, ya que la expresión literaria no funcionaría sin un referente pragmático. Por otra parte, todo texto de ciencia ficción está en contacto con la realidad futura por ser el resultado de una lectura referencial científica inscrita en lo temporal.

En conclusión, el relato de Chiang plantea una cuestión fundamental: la realidad ha dejado de tener una estabilidad temporal única, el tiempo humano es una convención, una construcción lingüística, un modelo narrativo creado por los seres humanos. Según la lingüística heptápoda que propone en su formato narrativo Chiang, la obra literaria se convierte en un mecanismo verbal que funciona en relación con la realidad exterior creando el universo lingüístico que remite a una realidad que se basa en su propia ficcionalidad. Se puede concebir entonces en la narrativa de la ciencia ficción de Chiang la existencia de una variación temporal que se define por su multidimensionalidad lingüística en concordancia con nuestra noción de realidad extratextual. Como se hace evidente, el relato de Chiang descansa sobre la problematización de esa visión arbitraria y, sin embargo, compartida de lo temporal. La 
poética de la ciencia ficción de Chiang no sólo evidencia la coexistencia temporal de lo posible y lo imposible dentro del universo habitado por humanos y heptápodos, sino también argumenta sobre el cuestionamiento de dicha coexistencia, por la propia lingüista protagonista, dando pruebas que esta podría existir dentro y fuera del texto. Cuando ya se hayan ido los heptápodos, Banks reflexiona: "Me hubiera gustado experimentar más la visión heptápoda del mundo, sentir como ellos se sentían. Entonces quizá podría sumergirme completamente en la necesidad de los acontecimientos, como deben de estar ellos, en lugar de chapotear en la orilla durante el resto de mi vida. Pero eso nunca sucederá (Chiang 2017: 121)."

Aunque la visión heptápoda del mundo no fuese sino una mirada desde la ficción literaria hacia la condición humana de sujeto lingüístico basada en las convenciones narrativas y en conocidas teorías lingüísticas y narratológicas, el texto de Chiang sugiere algunos conceptos interesantes. Los eventos sólo son, no pasan porque el presente no se mueve, ni pasa, sólo cambian los individuos. De ser así, para los seres humanos, el espacio-tiempo es la propia experiencia, el lugar de memoria o esperanza que en la medida de lo posible puede ser representado, reconstruido en la conciencia, creado e inventado en la ficción, almacenado en los libros. La inscripción en el tiempo condiciona la lengua, la cultura, la investigación científica y la expresión artística. Por una parte, el tiempo se mide en horas, meses y años, sistema ficticio que permite una representación del paso del tiempo a través de un movimiento en el espacio de un reloj. Por otra parte, el tiempo que marcan los relojes se mide en analogía con el movimiento en órbita de la Tierra. Por lo tanto, las categorías del movimiento espacial se conciben en su dimensión temporal. El trayecto está fragmentado en unidades temporales, por eso la representación espacial, por ejemplo, la distancia de un viaje se mide también en la medida del reloj como periodo del tiempo necesaria para ir de un lugar a otro. En realidad, representamos el movimiento lineal (espacio) en un sistema de medida circular (tiempo), mientras al mismo tiempo nos trasladamos en un espacio circular (Tierra) representando el trayecto en un sistema de tiempo lineal (pasado-futuro), unificando espacio y tiempo en nuestra experiencia de lo real y lo ficticio a través del lenguaje que articula lo material (sonidos, letras, textos) con lo inmaterial (ideas, memorias, planes). $\mathrm{Y}$ todo movimiento del uroboros de nuestras vidas está inscrito en nuestra memoria colectiva lineal, el lenguaje, una memoria compartida a-temporalmente $y$, sin embargo, vivida individualmente en forma de cronotopos en el espacio-tiempo propio del lector. Por consiguiente, para los humanos, el viaje en el tiempo sería posible gracias a las líneas de un texto durante la lectura, nuestra nave espacial es el lenguaje humano, oral o escrito. Así, el texto que narra la historia de la máquina del tiempo de $\mathrm{H}$. G. Wells, nada más que una idea codificada en lenguaje literario, si se lee existe en la conciencia lectora y en la cultura mundial. Si se trata de viajar en el tiempo, el lenguaje por la literatura nos pueda llevar a cualquier momento pasado o futuro porque es nuestra máquina de tiempo, los "bloques" heptápodos de recuerdos son nuestras bibliotecas donde todo el tiempo humano está experimentado como pasado y futuro a la vez en los volúmenes que cuentan las historias de nuestras vidas que nos quedan todavía por leer. Por eso, quizás sea la propia cultura la única manera de trasladarse por el continuum del espacio-tiempo; leyendo un libro sería viajar en lenguaje por el espaciotiempo, la memoria humana sería una literatura de viajes, recuerdos de viajes literarios. ¿Qué es la Odisea sino el viaje de regreso a Ítaca que se repite por milenios? El viaje de regreso al espaciotiempo del lenguaje homérico; El viaje simultáneo donde pensar en Heptápodo A y B es escribir y leer en lenguaje humano, enunciar el viaje significa realizarlo en el espacio-tiempo.

\section{BIBLIOGRAFÍA}

AGAR, M. (1994). Language shock: Understanding the culture of conversation. Nueva York: William Morrow \& Co.

BAJTIN, M.M. (1989). “Las formas de tiempo y del cronotopo en la novela. Ensayos de poética histórica". En Bajtin, Mijail. M: Teoría y estética de la novela. Madrid: Taurus, 237-409.

BOETSCH, P. (2003). "La literatura de viajes y la mirada antropológica", en Boletín de Literatura Comparada, Número Especial "Literatura de viajes", 49-627.

BORODITSKY, L. (2011). "How Languages Construct 
Time", en Stanislas Dehaene \& Elizabeth Brannon (ed.): Space, Time and Number in the Brain. Stanford, USA: Elsevier Inc.

CASASANTO, D., BORODITSKY, L., et al. (2004). "How deep are effects of language on thought? Time estimation in speakers of English, Indonesian, Greek, and Spanish", en K. Forbus, D. Gentner, \& T. Regier (eds.): Proceedings of the 26th Annual Conference of the Cognitive Science Society. Mahwah, NJ: Lawrence Erlbaum Associates, 186-191.

COLOMBI NICOLIA, B. (2006). "EI viaje y su relato", en Latinoamérica, 43, 11-35.

CONTURSI, M. E. \& FERRO, F. (2000). La Narración. Usos y teorías. Bogotá: Grupo Editorial Norma.

DE OTO, A. \& RODRÍGUEZ, J. (2010). "Travesías. Ensayo sobre el viaje, las imágenes y los desplazamientos", en Hermeneutic, 9, s.n.

DE PIENDA, J.A (2007). “Tiempo en Hawking. Crítica desde una perspectiva filosófica”, en Herrera Guevara, A. (ed.): De Animales y Hombres. Studia Philosophica. Madrid: Ediciones de la Universidad de Oviedo, 239-270.

FEYNMAN, R. (2002). Seis piezas fáciles. Barcelona: Crítica.

FrankLIN, H. B. (1995). Future Perfect. American Science Fiction of the Nineteenth Century. An Anthology. Nueva York: Rutgers: University Press.

FULGA, A. (2012).

"Language and the perception of Space, Motion and Time", en Concordia Working Papers in Applied Linguistics, 3, 26-37.

FUSELL, P. (1980). Abroad. British Literary Traveling Between the Wars. Nueva York: Oxford University Press.

GONZÁLEZSALVADOR, A. (1984). "De to fantástico y de la literatura fantástica", en Anuario de Estudios Filológicos, VII, 207-226.

GREENE, B. (2006). El universo elegante. Supercuerdas, dimensiones ocultas y la búsqueda de una teoría unificada. Barcelona: Crítica.

HAWKING, S. (1988). Historia del Tiempo: Del Big Bang a los Agujeros Negros. Barcelona: Editorial Crítica.

HICKMAN, M. (1987). Social and functional approaches to language and thought. Nueva York: Academic Press.

LEJEUNE, P. (1991). “El pacto autobiográfico”, en Anthropos, 29, 47-61.
MORENO, F. A. (2009). "La ficción prospectiva: propuesta para una delimitación del género de la ciencia ficción", en P. López Pellisa y F.A. Moreno (eds.), Ensayos sobre ciencia ficción y literatura fantástica, 62-65. Madrid: Universidad Carlos III de Madrid.

MINKOWSKI, H. (1908/9). "Raum und Zeit", en Jahresberichte der Deutschen MathematikerVereinigung, 18, 75-88.

NANDORFLY, M.J. (2001). "La literatura fantástica y la representación de la realidad", en Roas David (comp.), Teorías de lo fantastico, Madrid: Arco/Libros, 243-261.

PARRA, M. (1988). "La hipotesis Sapir-Whorf", en Forma y Función, 3, 9-16.

POEPPEL, E. (1978). "Time perception", en Richard Held et al. (eds.), Handbook of Sensory Physiology, Vol VIII: Perception. Berlin: SpringerVerlag, 713-729.

ROAS, D. (2001). "La amenaza de lo fantástico", en David Roas (comp.). Teorías de lo fantástico. Madrid: Arco/Libros, 7-46.

RODRÍGUEZ HILLÓN, D. V. (2015). “Acercamientos a la ciencia ficción”, en La Palabra, 27, 173-187.

RODRÍGUEZ PEQUEÑO, J. (2018). “Jules Verne: from the Odyssey to Science Fiction", en Castilla. Estudios de Literatura, 9, 1-19.

SAPIR, E. (1949). Culture, Language and Personality. Berkeley/Los Angeles: The regents of the University of California.

TODOROV, T. (1993). Las morales de la historia. Barcelona: Paidós.

UDÍAS VALLINA, A. (2014). "El tiempo: una cuestión siempre abierta", en Crítica, 990, 24-29.

WELLS, H. G. (2001), La máquina del tiempo y otros relatos. Madrid: Valdemar Editores.

WEYL, H. (1949). Philosophy of Mathematics and Natural Sciences. Princeton: Princeton University Press.

WHORF, B. (1956). Language, thought, and reality. Cambridge, Massachusetts: The M.I.T. Press.

ZUKAV, G. (1981). La danza de los maestros. Barcelona: Argos Vergara.

CRÍTICA N. 990 Marzo-Abril 2014 